
\title{
25 Research Soure \\ Effects of Sodium Pyruvate on Exercise-Induced Lactic Acidosis and Exercise Performance
}

\section{Yanping Yang}

Beijing Sport University https://orcid.org/0000-0002-2271-1896

Junqiang Qiu ( $\nabla$ qiujq001@sina.com )

Beijing Sport University https://orcid.org/0000-0001-8313-9216

\section{Mengyue Wang}

Beijing Sport University

Lin Feng

Beijing Sport University

\section{Dan Luo}

Beijing Sport University

\section{Ruirui Gao}

Beijing Sport University

\section{Fangqiang Zhou}

Fresenius Medical Care, Dialysis Centers in Chicago

\section{Research article}

Keywords: Exercise-induced lactic acidosis, High-intensity interval exercise, Energy metabolism, Exercise performance, Sodium pyruvate

Posted Date: April 21st, 2021

DOl: https://doi.org/10.21203/rs.3.rs-429807/v1

License: (c) (i) This work is licensed under a Creative Commons Attribution 4.0 International License. Read Full License 


\section{Abstract}

Background. The beneficial effects of pyruvate on lactic acidosis and energy metabolism have been studied. The ability to attenuate acidosis and improve aerobic system contribution are essential for team sports athletes to perform multiple sprints in a limited time. This study aimed to investigate the impact of pyruvate supplementation on energy metabolism and lactic acidosis during high-intensity interval exercise (HIIE) and to evaluate its role in repeated sprint exercise (RSE) performance.

Methods: Fourteen national-level male soccer athletes from China Football College (age: $20 \pm 2$ years, body fat: $13.11 \pm 3.50 \%$ ) participated in a randomized, double-blind, crossover study. Each subject underwent two HIIE and RSE tests after one week of supplementation with $0.1 \mathrm{~g} / \mathrm{kg} / \mathrm{d}$ of pyruvate or placebo. Venous blood gas $\left(\mathrm{pH}\right.$, bicarbonate $\left(\mathrm{HCO}_{3}{ }^{-}\right)$, base excess $(\mathrm{BE})$ and oxygen partial pressure $\left.\left(\mathrm{pO}_{2}\right)\right)$ were measured at baseline, pre-HIIE, post-HIIE, pre-RSE and post-RSE. Finger-stick capillary lactate were collected at baseline, immediately after each bout of HIIE, and 3, 5, 7 and 10 min post HIIE. The energy system contributions during HIIE and exercise performance of RSE were analyzed.

Results. Blood pH $(p<0.01), \mathrm{HCO}_{3}{ }^{-}(p<0.01)$ and $\mathrm{BE}(p<0.01)$ were significantly lower than their baseline levels at post-HIIE, pre-RSE and post-RSE in both Group pyruvate (PYR) and Group placebo $(\mathrm{PLA})$, respectively. Blood $\mathrm{pH}, \mathrm{HCO}_{3}{ }^{-}$and $\mathrm{BE}$ were significantly improved in PYR at pre-HIIE $(p<0.01)$, postHIIE $(p<0.01)$ and pre-RSE $(p<0.01)$ than in PLA. Furthermore, blood BE remained higher in PYR than in PLA until the end of RSE $(p<0.05)$. Blood $\mathrm{pO}_{2}$ was higher at post-HIIE $(p<0.05)$, pre-RSE $(p<0.01)$ and post-RSE $(p<0.01)$, compared to baseline, in two groups and further higher in PYR than in PLA at postHIIE $(p<0.05)$ and post-RSE $(p<0.05)$. During HIIE, the contribution of the aerobic system in the fourth bout of HIIE was higher in PYR than in PLA $(p<0.05)$. The contribution of the anaerobic energy was higher than that of the aerobic energy in PLA $(p<0.01)$, but without difference in PYR $(p>0.05)$. In addition, compared with PLA, the relative peak power (RPP) of the first and fifth sprints, the relative average power (RAP) of the fifth sprint and the average of RPP and RAP during RSE were significantly improved in PYR $(p<0.05)$. However, the blood lactate $(p>0.05)$, PD\% of each bout $(p>0.05)$ or average PD\% of RSE $(p>0.05)$ showed no significant difference between the two groups.

Conclusion: Pyruvate supplementation with $0.1 \mathrm{~g} / \mathrm{kg} / \mathrm{d}$ for a 1-week enhances aerobic metabolism and buffers exercise-induced metabolic acidosis but not lactic acidosis during HIIE, improving RSE performance in moderate acidosis. Thus, after 1 week pyruvate supplementation, athletes are expected to balance aerobic and anaerobic energy contributions and attenuate metabolic acidosis during team sports, improving exercise performance.

\section{Introduction}

Most team sports involve a number of high-intensity exercises and sprints with incomplete recovery intervals ${ }^{1}$. During high-intensity interval exercise (HIIE), the demand for ATP is greatly increased and exceeds the energy generation by aerobic metabolism. Therefore, the dependence on anaerobic 
(phosphagen and glycolysis) energy supply is promoted. The amount of hydrogen lon $\left(\mathrm{H}^{+}\right)$created by anaerobic metabolism is uncoupled with that eliminated with aerobic metabolism ${ }^{2}$. Intracellular $\mathrm{H}^{+}$is transported to the blood together with lactate. In the blood, $\mathrm{H}^{+}$is eliminated by bicarbonate $\left(\mathrm{HCO}_{3}{ }^{-}\right)$and

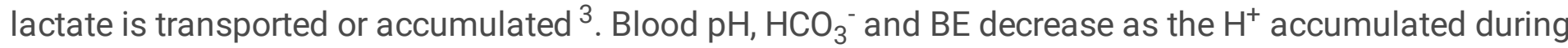
HIIE exceeds the maximum buffering potential and lactate accumulates simultaneously, resulting in intracellular and extracellular metabolic acidosis ${ }^{2}$. Exercise-induced metabolic acidosis is an important form of metabolic acidosis and is specifically classified into the category of lactic acidosis. Lactic acidosis described as the blood $\mathrm{pH}$ level $<7.35, \mathrm{HCO}_{3}{ }^{-}$level $<20 \mathrm{mmol} / \mathrm{L}$, base excess (BE) level $<-2$ $\mathrm{mmol} / \mathrm{L}$ and accompanied by the lactate level $>5 \mathrm{mmol} / \mathrm{L}^{4}$. The $\mathrm{pH}$ value of skeletal muscle could drop to 6.8 along with extracellular lactic acidosis in an intense soccer match ${ }^{5}$. Although the effect of lactic acidosis on exercise performance is controversial ${ }^{6,7}$, the large accumulation of $\mathrm{H}^{+}$in skeletal muscle may impair muscle contraction and exercise efficiency ${ }^{8}$.

For improving exercise performance, many studies have focused on the effect of alkaline buffers. Typical alkaline buffers mainly reduce extracellular acidosis by acting as physical and chemical buffers with limited metabolism ${ }^{9}$. On the other hand, pyruvate can be transferred into cells as an intracellular buffer to efficiently reduce lactic acidosis ${ }^{10,11}$. It mainly relies on regulating intracellular energy metabolism to reduce lactic acidosis additionally with the specific low dissociation constant of pyruvate $(\mathrm{pKa}=2.49)^{11}$, 12. Studies have shown that pyruvate is a crucial intermediate of glucose metabolism and a natural inhibitor of pyruvate dehydrogenase kinase (PDK), which improves pyruvate dehydrogenase (PDH) activity and benefits aerobic metabolism ${ }^{13,14,15}$. Moreover, reports have demonstrated that pyruvate can maintain glycolytic metabolism through its spontaneous reductive reaction by lactate dehydrogenase and improvement of key glycolytic enzymes ${ }^{11,14}$.

In fact, pyruvate is a superior buffer, as demonstrated in oral rehydration of shock that only can pyruvate reverse lactic acidosis, other than bicarbonate or citrate on an equimolar basis in shock resuscitation of animal models ${ }^{16}$. Previous studies found that the blood $\mathrm{pH}$ values were raised with reversal of hypoxic lactic acidosis after resuscitation by pyruvate administration in hemorrhagic shock models ${ }^{17,18}$. It was also discovered that pyruvate could boost blood $\mathrm{pH}$ and $\mathrm{BE}$ after reperfusion in a rat model of severe intestinal ischemia-reperfusion injury ${ }^{19}$. Moreover, perfused with hyperpolarized tracer $\left[1-{ }^{13} \mathrm{C}\right]$ pyruvate in the heart of rats, the production of $\mathrm{H}^{13} \mathrm{CO}_{3}{ }^{-}$was increased ${ }^{20}$. Particularly, pyruvate was reported to raise intracellular $\mathrm{pH}$ in failing human myocardium and to minimize hyperchloremic acidosis in a rat model ${ }^{10}$, 12 .

Sodium pyruvate is a safe substance $\left(\mathrm{LD}_{50}>10 \mathrm{~g} / \mathrm{kg} \text { oral pyruvate in rats }\right)^{21}$. With oral pyruvate treatment, the subjects reported no gastrointestinal symptoms with single doses less than $10 \mathrm{~g}^{21}$. Human studies have reported that single ingestion with $0.1 \mathrm{~g} / \mathrm{kg}$ pyruvate can increase the blood $\mathrm{pH}$, $\mathrm{HCO}_{3}{ }^{-}$and $\mathrm{BE}$ of healthy individuals at rest $60 \mathrm{~min}$ after intake and its buffering effect can last for 120 
$\min ^{22,23}$. However, acute supplementation with pyruvate seemed to be ineffective for high-intensity exercise-induced lactic acidosis ${ }^{23}$. Whether multiple pyruvate supplementation can further regulate the energy metabolism and attenuate lactic acidosis during exercise remains unknown.

Repeated sprint exercise (RSE) is generally considered to be the determining part of team sports performance despite the fact that sprinting activities account for only $10 \%$ of the total distance of team sports ${ }^{24}$. Team sports performance is affected by RSE fatigue factors, including insufficient creatine phosphate $(\mathrm{PCr})$ resynthesis and intramuscular $\mathrm{H}^{+}$accumulation ${ }^{25}$. Therefore, reducing lactic acidosis and increasing the potential of the aerobic metabolism to resynthesize PCr are of great benefits in the performance of team sports athletes.

To our knowledge, few studies have documented the effects of multiple sodium pyruvate supplementation this topic. This present study evaluated the role of oral sodium pyruvate on exerciseinduced lactic acidosis and team sport performance from the perspective of energy metabolism. We hypothesized that multiple pyruvate ingestion would contribute to energy availability and environmental alkalization during RSE, leading to better performance.

\section{Methods}

\section{Subjects}

Fifteen national-level male soccer athletes from China Football College volunteered to participate in this study. Exclusion criteria included: 1) hypertension, diabetes, cardiovascular risk factors and any other diagnosed metabolic disorders (e.g., acid-base imbalance); 2) smoking, ingested alcohol, caffeine and any other ergogenic supplements (e.g., sodium bicarbonate and creatine). Inclusion criteria: 1) age 18-24 years; 2) BMI ranging from 18.5 to $23.9 \mathrm{~kg} / \mathrm{m}^{2}$; and 3) at least 5 years of regular soccer training experience with maximal oxygen uptake over $50 \mathrm{ml} / \mathrm{kg} / \mathrm{min}$. The study protocol was approved by the Internal Review Board of Beijing Sport University (2020057H). All subjects were informed of the study aims and were asked to sign an informed consent form. One participant dropped out after the graded cycling exercise test. Fourteen athletes completed all the sessions and were included in the data analyses (Table 1).

\section{Study design}

Randomized, double-blind, crossover trials were used in this research. There were five laboratory visits, each separated by 7 days. The first visit involved personal information collection, body composition measurement, familiarization with the exercise test protocol, and performed a graded cycling exercise test (GXT). In the second visit, participants took sodium pyruvate (PYR) or maltodextrin (Placebo, PLA) (0.1 $\mathrm{g} / \mathrm{kg} / \mathrm{d})$ for 7 days parallelly and randomly in PYR $(\mathrm{n}=7)$ and PLA $(n=7)$, respectively. During the third visit, $45 \mathrm{~min}$ after the last supplementation, the subjects took a 15 -min resting oxygen uptake test $\left(15-\mathrm{RO}_{2}\right)$, performed high-intensity interval exercise (HIIE) test to induce lactic acidosis, and collected a 6-min 
oxygen uptake immediately after HIIE (6-VO $\left.\mathrm{VO}_{2}\right)$. An RSE test was performed 10 min after HIIE. In the fourth visit, participants who were supplied with pyruvate/maltodextrin in the second visit were switched to maltodextrin/pyruvate, parallelly and randomly in PYR $(n=7)$ and PLA $(n=7)$. After a 1-week, they returned to the laboratory and repeated the tests they had done during the third visit. Each subject performed the tests at the same time of day and in the same laboratory environment.

Participants arrived in a resting and thoroughly hydrated condition following $\geq 2 \mathrm{~h}$ postprandial, avoiding any strenuous exercise and consumption with carbonate, alcohol and caffeine $24 \mathrm{~h}$ before each exercise test. A 3-day dietary recall was used to measure calorie intake and macronutrient consumption (2 weekdays and 1 weekend). Subjects used a 24-h recall to measure dietary consumption the day before the first trial and were asked to replicate the same diet as accurately as possible in subsequent studies. To ensure compliance, participants were expected to ingest the supplement under supervision. Fig. 1 presents a timeline for this study.

\section{Body composition measurements}

A calibrated electronic scale was used to calculate height to the nearest $0.1 \mathrm{~cm}$ (GMCS-SGJ3, Jianmin, Beijing, China). Body composition was assessed $2 \mathrm{~h}$ after a meal by multifrequency bioelectrical impedance measurement device (Inbody 230, Biospace, Seoul, Korea).

\section{Graded cycling exercise test (GXT)}

Each subject underwent GXT on an electromagnetically braked cycle ergometer (EC 3000e, CUSTO Med, Ottobrunn, Germany) to determine peak power $\left(\mathrm{W}_{\max }\right)$ and maximum oxygen uptake $\left(\mathrm{VO}_{2 \mathrm{max}}\right)$.

Participants adjusted the seat and warmed up for $3 \mathrm{~min}$ at $50 \mathrm{~W}$. Then, the pedal frequency was kept between 75-80 rpm and the load was increased at $30 \mathrm{~W} / \mathrm{min}$. The subjects' standards for exhaustion included the following: 1) Failed to keep $75 \mathrm{rpm}$ for $5 \mathrm{~s} ; 2$ ) Increased the power load with the oxygen uptake $\left(\mathrm{VO}_{2}\right)$ rise $\left.\leq 150 \mathrm{ml} / \mathrm{kg} / \mathrm{min} ; 3\right)$ Respiratory exchange rate $\left(\mathrm{VCO}_{2} / \mathrm{VO}_{2}\right) \geq 1.10$ and 4) Heart rate reached 220 -age. $\mathrm{VO}_{2 \max }$ is the average oxygen consumption $30 \mathrm{~s}$ before reaching exhaustion ${ }^{26}$. $\mathrm{W}_{\max }$ is determined as the power of the last completed stage plus the fraction of time spent in the final uncompleted stage multiplied by $30 \mathrm{~W}^{27}$. During the test, the Borg scale (6-20) ${ }^{28}$ was used to determine the rate of perceived exertion (RPE) and Polar V800 (Polar Electro Oy, Oulu, Finland) was used to measure heart rate.

\section{Supplementation protocol}

Sodium pyruvate in high quality (Lianlu industrial Co., Ltd., Shanghai, China) and maltodextrin were randomly packed in capsules A or B; both were similar in appearance, size and weight. Each participant took $0.1 \mathrm{~g} / \mathrm{kg} / \mathrm{d}^{23}$ pyruvate or maltodextrin for 7 days (ingested 2 capsules just after a meal and 1 capsule before sleep on the first 6 days; ingested all the remaining capsules $60 \mathrm{~min}$ prior to the exercise test on the 7 th day ${ }^{23}$ ). Subjects took the capsule A were switched to the capsule B and vice versa during the test timeline. 


\section{Oxygen uptake test}

$\mathrm{VO}_{2}$ was measured with breath-by-breath at $\mathrm{GXT}, 15-\mathrm{RO}_{2}, \mathrm{HIIE}$ test and $6-\mathrm{VO}_{2}$, using a portable gas analyzer (Cortex Metamax 3B, CORTEX Biophysik, Leipzig, Germany). Participants took a break in a sitting position to measure $15-\mathrm{RO}_{2}$ and $6-\mathrm{VO}_{2}$. The $\mathrm{RO}_{2}$ was determined by using the average of the last 10 min of data collected ${ }^{29}$. The gas analyzer was calibrated before each test in accordance with the manufacturer's instructions.

\section{HIIE protocol}

HIIE was conducted in an electromagnetically braked cycle (Ergoline Ergoselect 100K, Ergoline, Bitz, Germany). During the test, subjects took a 5-min warm-up at $60 \mathrm{~W}$. HIIE consisted of $4 \times 1 \mathrm{~min}$ highintensity cycling at $110 \% \mathrm{~W}_{\max }$ and separated by 1 min rest. Cadence was constant $(90-100 \mathrm{rpm}$ ) during high-intensity bout. Subjects remained seated on the ergometer, taking a 10-min break after finishing the last bout ${ }^{30}$.

\section{RSE test}

RSE consisting of $6 \times 6 \mathrm{~s}$ of all out maximal cycling was performed on a mechanically braked cycle ergometer (894E, Monark, Vansbro, Sweden). Exercise load equaled weight (kg) $\times 0.087 \mathrm{kp} / \mathrm{kg}$ (weight) ${ }^{31}$. During the test, participants adjusted the seat and began to fully ride following the staff countdown " 3,2 , 1 , start". Once the cadence reached $110 \mathrm{rpm}$, the load of inertial ergometers was adjusted to the predetermined load. Subjects kept fully pedaling to complete a $6 \mathrm{~s}$ cycling exercise and encouragement was provided to enable subjects to exert maximum ability. Each sprint had a $24 \mathrm{~s}$ rest interval ${ }^{25}$.

Relative peak power (RPP): the highest power output (PP) relative to body mass observed in each $6 \mathrm{~s}$; Relative average power (RAP): the average power output (AP) relative to body mass maintained in each 6 s; Power drop (PD\%): the percentage of mechanical power decay over the period of the test relative to the peak power, according to the following equation: $P D \%=[(P P-A P) / P P \times 100]^{25}$. All the parameters were calculated via Monark Anaerobic Testing software (Version: 3.3.0.0, developed in cooperation with HUR Labs).

\section{Blood collection and analyses}

Capillary blood samples with $10 \mu \mathrm{l}$ of fingerstick were collected (wiped away the first drop of blood) in a Biosen capillary tube (EKF Diagnostics, Barleben, Germany) at baseline, the end of each bout of HIIE, and 3, 5, 7 and 10 min after HIIE. The samples were used to measure blood lactate concentrations with a lactate analyzer (Biosen C-Line, EKF Diagnostics, Barleben, Germany).

Blood samples of $1.0 \mathrm{ml}$ were obtained from the ulnar vein at baseline, pre-HIIE, post-HIIE, pre-RSE and post-RSE. The samples were collected in sodium heparin tubes (YA1430, Solarbio, Beijing, China) and immediately assessed for blood $\mathrm{pH}, \mathrm{HCO}_{3}{ }^{-}, \mathrm{BE}$ and oxygen partial pressure $\left(\mathrm{pO}_{2}\right)$ by a blood gas analyzer 
(Radiometer ABL80, FLEX CO-OX, Willich, Germany). Additionally, the blood $\mathrm{HCO}_{3}{ }^{-}$was calculated from the partial pressure of carbon dioxide and $\mathrm{pH}$ values according to the Henderson-Hasselbalch equation and the blood $\mathrm{BE}$ was calculated from $\mathrm{HCO}_{3}{ }^{-}$and hemoglobin $(\mathrm{Hb})$ determined by gas analyzer according

to the following equation ${ }^{32}$ :

$$
B E=(1.0-0.0143 * \mathrm{Hb}) *\left[\left(\mathrm{HCO}_{3}^{-}\right)-24.8+(7.7+1.43 * \mathrm{Hb}) *(\mathrm{pH}-7.4)\right]
$$

\section{Estimation of energy contribution}

The aerobic energy contribution was estimated by subtracting the resting oxygen consumption from the oxygen consumption obtained during each $110 \% \mathrm{~W}_{\max }$ bout. The consumed oxygen was transferred to energy. One liter $\mathrm{O}_{2}$ is converted into $20.92 \mathrm{~kJ}$ energy equivalent ${ }^{33}$. The lactate difference before and after each $110 \% \mathrm{~W}_{\max }$ bout and the lactate accumulated during HIIE were assumed to calculate the glycolytic energy contribution of each bout and total HIIE, respectively. The accumulation of $1.0 \mathrm{mmol} / \mathrm{L}$ lactate corresponds to $3.0 \mathrm{ml} \mathrm{O}_{2} / \mathrm{kg}$ of body weight ${ }^{33}$. The phosphagen energy system contains two sections. First, resting oxygen uptake was subtracted from the oxygen consumption obtained during each 1-min interval. Second, fast component of excess post-exercise oxygen uptake (EPOC fast) accessed during $6-\mathrm{VO}_{2}$ was adjusted by a biexponential model. The EPOC fast is assumed to be the product of the amplitude and time constant of the first exponential model (OriginPro 8.0, OriginLab, Microcal, Massachusetts, USA) ${ }^{34}$.

\section{Statistical analyses}

A power analysis (Power 1- $\beta=0.9$ and $\alpha=0.05$ ) was performed with a priori in $G *$ Power software version 3.1.9.6 (Universitat Kiel, Germany), and at least 12 participants were needed for the present study. Data analyses were carried out by using SPSS, version 22.0 (SPSS Inc. Chicago, IL, USA). The Shapiro-Wilk test was used to verify the assumptions of normality of the data. A non-parametric Kruskal-Wallis test was used to analyze the power drop (\%) of each sprint and Dunn-Bonferroni test as a post-hoc test. Two-way repeated-measures ANOVA was performed to assess the interaction between time (baseline, each bout of HIIE and each sprint of RSE) and the cohort (PYR and PLA). Fisher's least square significant difference (LSD) post-hoc analyses were performed when significant interactions were observed. Independent samples t-tests were used to measure relative energy contribution during HIIE and average RPP, RAP and $P D \%$ during RSE. Values were expressed as the mean \pm SD or median (P25, P75). The statistical significance level was set at $p<0.05$.

\section{Results}

All subjects were supplemented with pyruvate or placebo as required and under the supervision of research staff. There were no side effects reported by the participants with the supplementation and there was no significant effect of treatment order in the research. During the study, participants maintained their typical eating habits and did not change their physical activity patterns. 


\section{Exercise induced lactic acidosis}

Table 2 shows the venous blood $\mathrm{pH}, \mathrm{HCO}_{3}{ }^{-}, \mathrm{BE}$ and lactate for each group with no significant different at baseline. In PYR, blood $\mathrm{HCO}_{3}{ }^{-}(p<0.01)$ and $\mathrm{BE}(p<0.01)$ were significantly increased at pre-HIIE compared with baseline. Blood $\mathrm{pH}(p<0.01), \mathrm{HCO}_{3}{ }^{-}(p<0.01)$ and $\mathrm{BE}(p<0.01)$ were significantly decreased, while blood lactate $(p<0.01)$ was significantly increased at post-HIIE, pre-RSE and post-RSE compared with baseline in each group. Compared to $\mathrm{PLA}$, blood $\mathrm{pH}, \mathrm{HCO}_{3}{ }^{-}$and $\mathrm{BE}$ were significantly improved in PYR at pre-HIIE $(p<0.01)$, post-HIIE $(p<0.05)$ and pre-RSE $(p<0.05)$, Moreover, blood BE in PYR was higher than PLA until post-RSE $(p<0.05)$.

\section{Energy contribution}

Figure $2 \mathrm{~A}-2 \mathrm{C}$ display the energy contribution during HIIE for each group. In both groups, compared to bout 1 , aerobic energy system contribution of bouts $2-4(p<0.01)$ and the phosphagen energy system contribution of bouts $2-3(p<0.01)$ were significantly increased, while the glycolytic energy system contribution of bouts $2-4(p<0.01)$ showed a significant decrease. Compared with PLA, the aerobic energy system contribution of the fourth bout $(p<0.05)$ was significantly improved in PYR. However, the contributions of glycolytic and phosphagen energy systems were not significantly changed between PYR and PLA during the four bouts of high-intensity exercise $(p>0.05)$.

Figure 2D shows the percentage of aerobic and anaerobic energy contributions during HIIE. There was no significant difference in the ratio of aerobic $(p>0.05)$ or anaerobic $(p>0.05)$ energy contributions during HIIE between PYR and PLA. But the ratio of the anaerobic energy contribution during HIIE was higher than that of aerobic energy in PLA $(48.07 \% \pm 3.22 \%$ vs. $51.93 \% \pm 3.22 \%, p<0.01)$, but no difference was observed in PYR $(49.47 \% \pm 1.77 \%$ vs. $50.53 \% \pm 1.77 \%, p>0.05)$.

Figure 3 displays the venous blood $\mathrm{pO}_{2}$ response during tests. Compared with baseline, the blood $\mathrm{pO}_{2}$ increase at post-HIIE $(p<0.05)$, pre-RSE $(p<0.01)$ and post-RSE $(p<0.01)$ in both groups. Compared with PLA, the blood $\mathrm{pO}_{2}$ significantly improved in PYR at post-HIIE $(p<0.05)$ and post-RSE $(p<0.05)$.

\section{RSE performance}

Compared to PLA, pyruvate significantly increased the average $\operatorname{RPP}(p<0.05)$ and $\operatorname{RAP}(p<0.05)$ but the average PD\% was not changed ( $p>0.05$ ) during RSE (Fig. 4A).

Figure 4B-4D display the RPP, RAP and PD\% during RSE for each sprint. In PYR, compared to sprint 1, the RPP of sprints $3-4$ and sprint $6(p<0.05)$, the RAP of sprint $3(p<0.05)$ and sprints $4-6(p<0.01)$ were decreased, while the PD\% of sprint $4(p<0.05)$ and sprints 5-6 $(p<0.01)$ were increased during RSE. In PLA, compared to sprint 1 , the RPP of sprint $5(p<0.05)$ and the RAP of sprints $4-6(p<0.05)$ were decreased, while the PD\% of sprints 4-6 $(p<0.01)$ were increased during RSE. Compared to PLA, the RPP 
of the first and fifth sprints $(p<0.05)$ and the RAP of the fifth sprint $(p<0.05)$ were significantly improved in PYR. There was no significant difference in the PD\% of each sprint during RSE between PYR and PLA.

\section{Discussion}

This was the first study to explore the effects of sodium pyruvate supplementation for 1 week on energy metabolism and lactic acidosis during HIIE and the RSE performance of well-trained soccer players. The same doses of pyruvate and maltodextrin were supplemented to eliminate the caloric interference factors. Further, we used two independent exercise programs in the investigation: equal bouts of HIIE for inducing lactic acidosis and estimating energy metabolism and RSE for evaluating exercise performance. The results demonstrated that the strenuous exercise generated severe lactic acidosis, as showed with the robust decrease of blood $\mathrm{pH}, \mathrm{HCO}_{3}{ }^{-}$and $\mathrm{BE}$ with increase of blood lactate in the two groups (Table 2), which was in consistence with previous findings 5,30 . Present data first showed that the multiple pyruvate supplements significantly reduced the imbalance between aerobic and anaerobic metabolisms and attenuated metabolic acidosis during HIIE. Furthermore, the RPP of the first and fifth sprints, the RAP of the fifth sprint and the average RPP and RAP during RSE were significantly increased in PYR. However, blood lactate, energy contribution of phosphagen and glycolytic energy systems during HIIE as well as PD\% of RSE had no significant change by pyruvate intake within this protocol.

One of main findings is that pyruvate supplementation for a 1-week elevated the blood buffering capacity before exercise, which was similar to the single acute supplementation protocol ${ }^{22,23}$. Another main finding suggested that one week of pyruvate supplementation increased the alkaline reserves and buffered HIIE-induced metabolic acidosis in a certain extent. In contrast, single pyruvate supplementation could not buffer lactic acidosis induced by $6 \mathrm{~min}$ high-intensity $\left(90 \% \mathrm{VO}_{2 \max }\right)$ exercise ${ }^{23}$. These data demonstrated that multiple pyruvate supplementation may play a better role in attenuating exerciseinduced metabolic acidosis than single acute supplementation, as shown that the present protocol boosted the aerobic metabolism and the $\mathrm{HCO}_{3}{ }^{-}$production during intense exercise. The $\mathrm{H}^{+}$was eliminated intracellularly and the efflux into blood was reduced ${ }^{35}$. In addition, it was also found that pyruvate bridged the gap between the aerobic and anaerobic energy contributions during HIIE (Fig. 3D). As a result, pyruvate eliminated the difference of $\mathrm{H}^{+}$generated by anaerobic metabolism and removed by aerobic metabolism, reducing the net accumulation of $\mathrm{H}^{+}$and alleviating exercise-induced metabolic acidosis $^{2}$.

Our finding also provided the direct evidence that the venous blood $\mathrm{pO}_{2}$ gradually increased and blood oxygen saturation $\left(\mathrm{SpO}_{2}\right.$ ) remained $96.5 \%-98 \%$ (data not showed) along with HIIE and RSE in soccer athletes, suggesting that sufficient oxygen was obtained to support aerobic metabolism ${ }^{36}$. As observed in previous study, the $\mathrm{pO}_{2}$ of earlobe arterialized blood was significantly increased after progressive exhaustive exercise ${ }^{37}$. These results strongly supported the concept that severe lactic acidosis could be caused by an imbalance of aerobic and anaerobic metabolism other than lack of oxygen during high- 
intensity exercise ${ }^{38}$. Accordingly, strenuous exercise induces metabolic, instead of hypoxic, lactic acidosis in this study, as demonstrated previously ${ }^{38}$. Furthermore, current research first demonstrated pyruvate significantly increased the venous blood $\mathrm{pO}_{2}$ at post-HIIE and post-RSE in PYR relative to PLA (Fig. 3). According to previous works, pyruvate was substantiated to improve the glycolytic energy generation and oxygen-carrying capacity of red blood cells during bypass surgery and storage, in vitro 39 , 40 . Pyruvate improved the blood $\mathrm{pO}_{2}$ during intestinal ischemia reperfusion, reducing ischemia reperfusion injury in rats ${ }^{41}$. A small dose of pyruvate in cardioplegia for cardiac bypass surgery showed robust cardioprotection although there is no data that pyruvate effects on the normal human heart during exercise ${ }^{42}$. Moreover, intravenous or oral pyruvate multi-organ protection of metabolism and function, including brain, heart, liver, kidney and intestine with preservation of visceral blood flow was reproducibly demonstrated in shock resuscitation of various animal models ${ }^{43}$. Therefore, present data strongly suggested that oral pyruvate in this protocol probably facilitated to benefit cardiac function, oxygencarrying capacity and systemic hemodynamics during the strenuous exercise.

According to the results, the energy supply of aerobic and phosphagen systems increased, while the glycolytic system decreased along with HIIE test in both groups (Fig. 2). These results were compatible with data from other literature on HIIE energy metabolism ${ }^{30}$. We also discovered that pyruvate enhanced the aerobic energy contribution in the fourth bout of HIIE. Moreover, pyruvate eliminated the gap between aerobic and anaerobic energy contributions during HIIE (Fig. 3D). As shown in previously, pyruvate activated PDH activity and promoted mitochondrial aerobic metabolism and produced more NADH and ATP 14,15 . Thus, pyruvate may increase the activity of the key rate-limiting enzymes of aerobic metabolism, such as PDH, balancing the gap between anaerobic and aerobic metabolisms during intense exercise.

Our study showed no significant differences in blood lactate and glycolytic metabolism between two groups during HIIE. These results may be superficially inconsistent with previous findings, as prior research demonstrated that pyruvate could alleviate blood lactate by promoting aerobic metabolism while protecting glycolytic metabolism ${ }^{11,40}$. Moreover, single pyruvate supplementation also demonstrated its protection effect on glycolytic metabolism during high-intensity exercise, however, which showed a significant lactate rise post-exercise ${ }^{23}$. One possible explanation for the absence of further lactate increase in PYR was that pyruvate promoted aerobic energy metabolism and consumed more lactate in mitochondrial oxidative phosphorylation during the interval period of HIIE, as previously shown ${ }^{10,44}$. In addition, pyruvate consumed intracellular $\mathrm{H}^{+}$and decreased the muscle-to-blood $\mathrm{H}^{+}$ gradient, reducing lactate output into blood ${ }^{35}$. Alternatively, the current pyruvate protocol just partially attenuated lactic acidosis induced by intense exercise. No further decease of blood lactate in PYR relative to PLA may indicate the insufficient pyruvate dose absorbed in this protocol.

Studies have shown that athletes often need hundreds of short-term sprints to ensure the possession and shooting rate during a team sports game ${ }^{45}$. The repeated sprint ability is mainly determined by the 
resynthesis of $\mathrm{PCr}$ and the removal ability of $\mathrm{H}^{+}$during the interval period ${ }^{8,25}$. However, as a match progresses, the consumption of $\mathrm{PCr}$ and the accumulation of $\mathrm{H}^{+}$limited the repeated sprint ability of athletes of high or ordinary levels ${ }^{8,46}$. In line with previous studies, the RPP and RAP declined and PD\% increased throughout each sprint during RSE in both groups, indicating a decrease in RSE efficiency 47 . However, compared to PLA, the RPP of the first $(+11.41 \%)$ and fifth $(+14.92 \%)$ sprints, the RAP of the fifth $(+10.72 \%)$ sprint and the average RPP $(+7.06 \%)$ and RAP $(+5.56 \%)$ during RSE were significantly increased in PYR. Therefore, pyruvate may enhance the ability of aerobic metabolism, facilitate to $\mathrm{PCr}$ resynthesis and attenuate the severe metabolic acidosis induced by exercise, significantly improving RSE performance. Present results provide additional evidence that the decline of exercise capacity is mostly associated with the severity of metabolic acidosis, rather than the blood lactate level.

Acidosis and dehydration are common events in intense soccer games, which have become critical factors that limit exercise performance ${ }^{5}$. Considering recent reports with pyruvate-enriched oral rehydration salt (Pyr-ORS) in effective treatments of hypoxic lactic acidosis in shock resuscitation or diabetes ${ }^{14}$, it is necessary to explore the effect of Pyr-ORS in intense team sports, which may enhance the oral pyruvate absorption and provide further benefits in lactic acidosis correction and exercise performance ${ }^{48}$. Further research should examine the dose-effect relationship between effective blood pyruvate levels and exercise.

\section{Limitations}

The participants were college soccer athletes. Due to training commitments, collecting muscle tissue for assessments of PCr, ATP and PDH was challenging. The present study evaluated the acid-base response by using the venous blood; if the arterial blood samples had been used, the effects of pyruvate on skeletal muscle cells during exercise could have been more efficiently evaluated.

\section{Conclusions}

Intensive exercise induced severe lactic acidosis caused by the imbalance of anaerobic metabolism in cytosol and aerobic metabolism in mitochondria, rather than systemic hypoxia. Supplementation with 0.1 $\mathrm{g} / \mathrm{kg} / \mathrm{d}$ pyruvate for one week significantly attenuated the severe metabolic acidosis and improve the balance of energy metabolic contributions during HIIE, which are beneficial in the RSE performance. However, the dosage of current pyruvate supply was not sufficient to significantly reduce exercise-induced lactic acidosis to an ideal extent. To increase the pyruvate supplementation dose efficiently absorbed may further improve pyruvate benefits in exercise performance.

\section{Abbreviations}


ATP: Adenosine triphosphate; AP: Average power output; BE: Base excess; Bout: High-intensity exercise bout; EPOC fast: Fast component of excess post-exercise oxygen consumption; GXT: Graded cycling exercise test; $\mathrm{H}^{+}$: Hydrogen ion; $\mathrm{Hb}$ : Hemoglobin; $\mathrm{HCO}_{3}{ }^{-}$: Bicarbonate; HIIE: High-intensity interval exercise; HR: Heart rate; NADH, Nicotinamide adenine dinucleotide; PD\%: Power drop\%; PDH: Pyruvate dehydrogenase; PLA: Group placebo; PDK, Pyruvate dehydrogenase kinase; PLA: Group placebo; pKa: Dissociation constant; $\mathrm{pO}_{2}$ : Oxygen partial pressure; Pre-HIIE: Pre-high-intensity interval exercise; Pre-RSE: Pre-repeated sprint exercise; Post-HIIE: Post-high-intensity interval exercise; Post-RSE: Post-repeated sprint exercise; PP: Peak power output; PYR: Group pyruvate; RPE: Rate of perceived exertion; RSE: Repeated sprint exercise; RAP: Relative average power; RPP: Relative peak power; $\mathrm{VO}_{2 m a x}$ : maximum oxygen uptake; $\mathrm{VO}_{2}$ : oxygen uptake; $\mathrm{W}_{\text {max }}$ : Maximal power output; $\mathrm{S}$ : Sprint exercise; $15-\mathrm{RO}_{2}$ : Resting oxygen uptake test; 6- $\mathrm{VO}_{2}$ : oxygen uptake test 6-min immediately after HIIE.

\section{Declarations}

\section{Ethics approval and consent to participate}

The research proposal was approved by the Internal Review Board of Beijing Sport University (BSU IRB). All participants signed written informed consent prior to participating in this study (2020057H).

\section{Consent for Publication}

Not applicable, no individual person's data was presented.

\section{Availability of data and material}

The datasets generated and/or analyzed as part of the current study are not publicly available due to confidentiality agreements with subjects. However, they can be made available solely for the purpose of review and not for the purpose of publication from the corresponding author upon reasonable request.

\section{Competing interests}

No authors declare competing interests.

\section{Funding}

Funding was provided by the Herbalife Winter Sports Development Fund and the National Key R\&D Program of China (2018YFC2000600).

\section{Authors Contributions}

YY was responsible for the study design, data collection, data interpretation, writing and revision of the manuscript under the supervision of JQ, who finally evaluated the manuscript. MW was in charge of participant recruitment and management. FZ shared the idea with JQ and YP, assisted data analysis and 
improved manuscript, DL, LF, and RG assisted in the completion of the manuscript. The authors declare no conflicts of interest with the current publication, and all authors approved the final version of the manuscript.

\section{Acknowledgments}

The authors would like to sincerely thank the participants for volunteering to participate in this study.

Note: The opinions or assertions contained herein are not a reflection of the view of Fresenius Medical Care, Dialysis Centers in Chicago, Illinois.

\section{References}

1. Spencer M, Bishop D, Dawson B, et al. Physiological and metabolic responses of repeated-sprint activities:specific to field-based team sports [J]. Sports Med. 2005;35(12):1025-44.

2. Robergs RA, Ghiasvand F, Parker D. Biochemistry of exercise-induced metabolic acidosis [J]. Am J Physiol Regul Integr Comp Physiol. 2004;287(3):R502-16.

3. Juel C, Klarskov C, Nielsen JJ, et al. Effect of high-intensity intermittent training on lactate and $\mathrm{H}+$ release from human skeletal muscle [J]. Am J Physiol Endocrinol Metab. 2004;286(2):E245-51.

4. Kamel KS, Oh MS, Halperin ML. L-lactic acidosis: pathophysiology, classification, and causes; emphasis on biochemical and metabolic basis [J]. Kidney Int. 2020;97(1):75-88.

5. Krustrup P, Mohr M, Steensberg A, et al. Muscle and blood metabolites during a soccer game: implications for sprint performance [J]. Med Sci Sports Exerc. 2006;38(6):1165-74.

6. Debold EP, Beck SE, Warshaw DM. Effect of low pH on single skeletal muscle myosin mechanics and kinetics [J]. Am J Physiol Cell Physiol. 2008;295(1):C173-9.

7. Overgaard K, Højfeldt GW, Nielsen OB. Effects of acidification and increased extracellular potassium on dynamic muscle contractions in isolated rat muscles [J]. J Physiol. 2010;588(Pt 24):5065-76.

8. Maclntosh BR, Holash RJ, Renaud JM. Skeletal muscle fatigue-regulation of excitation-contraction coupling to avoid metabolic catastrophe [J]. J Cell Sci. 2012;125(Pt 9):2105-14.

9. Lancha Junior AH, Painelli Vde S, Saunders B, et al. Nutritional Strategies to Modulate Intracellular and Extracellular Buffering Capacity During High-Intensity Exercise [J]. Sports Med. 2015;45(Suppl 1):71-81.

10. Yang J, Zhao JX, Wang Y, et al. Effects of sodium pyruvate on ameliorating metabolic acidosis [J]. Artif Cells Nanomed Biotechnol. 2016;44(1):48-55.

11. Zhou FQ. Pyruvate in the correction of intracellular acidosis: a metabolic basis as a novel superior buffer [J]. Am J Nephrol. 2005;25(1):55-63.

12. Hasenfuss G, Maier LS, Hermann HP, et al. Influence of pyruvate on contractile performance and $\mathrm{Ca}(2+)$ cycling in isolated failing human myocardium [J]. Circulation. 2002;105(2):194-9. 
13. Kukimoto-Niino M, Tokmakov A, Terada T, et al. Inhibitor-bound structures of human pyruvate dehydrogenase kinase 4 [J]. Acta Crystallogr D Biol Crystallogr. 2011;67(Pt 9):763-73.

14. Zhang XM, Deng H, Tong JD, et al. Pyruvate-Enriched Oral Rehydration Solution Improves Glucometabolic Disorders in the Kidneys of Diabetic db/db Mice [J]. J Diabetes Res, 2020, 2020: 2817972.

15. Sharma P, Walsh KT, Kerr-Knott KA, et al. Pyruvate modulates hepatic mitochondrial functions and reduces apoptosis indicators during hemorrhagic shock in rats [J]. Anesthesiology. 2005;103(1):6573.

16. Liu R, Hu XH, Wang SM, et al. Pyruvate in oral rehydration salt improves hemodynamics, vasopermeability and survival after burns in dogs [J]. Burns. 2016;42(4):797-806.

17. Hu S, Bai XD, Liu XQ, et al. Pyruvate Ringer's solution corrects lactic acidosis and prolongs survival during hemorrhagic shock in rats [J]. J Emerg Med. 2013;45(6):885-93.

18. Flaherty DC, Hoxha B, Sun J, et al. Pyruvate-fortified fluid resuscitation improves hemodynamic stability while suppressing systemic inflammation and myocardial oxidative stress after hemorrhagic shock [J]. Mil Med. 2010;175(3):166-72.

19. Petrat F, Rönn T, de Groot H. Protection by pyruvate infusion in a rat model of severe intestinal ischemia-reperfusion injury [J]. J Surg Res. 2011;167(2):e93-101.

20. Schroeder MA, Cochlin LE, Heather LC, et al. In vivo assessment of pyruvate dehydrogenase flux in the heart using hyperpolarized carbon-13 magnetic resonance [J]. Proc Natl Acad Sci U S A. 2008;105(33):12051-6.

21. Petkova I, Hristov V, Petrov K, et al. Oral application of sodium pyruvate in healthy persons and patients with diabetes mellitus type I [J]. Comptes Rendus de L'Academie Bulgare des Sciences, 2007, 60: $579-84$.

22. Olek RA, Luszczyk M, Kujach $S$, et al. Single pyruvate intake induces blood alkalization and modification of resting metabolism in humans [J]. Nutrition. 2015;31(3):466-74.

23. Olek RA, Kujach S, Wnuk D, et al. Single sodium pyruvate ingestion modifies blood acid-base status and post-exercise lactate concentration in humans [J]. Nutrients. 2014;6(5):1981-92.

24. Carling C, Bloomfield J, Nelsen L, et al. The role of motion analysis in elite soccer: contemporary performance measurement techniques and work rate data [J]. Sports Med. 2008;38(10):839-62.

25. Girard O, Mendez-Villanueva A, Bishop D. Repeated-sprint ability - part I: factors contributing to fatigue [J]. Sports Med. 2011;41(8):673-94.

26. Jacobs RA, Flück D, Bonne TC, et al. Improvements in exercise performance with high-intensity interval training coincide with an increase in skeletal muscle mitochondrial content and function [J]. J Appl Physiol (1985). 2013;115(6):785-93.

27. Wang J, Qiu J, Yi L, et al. Effect of sodium bicarbonate ingestion during 6 weeks of HIIT on anaerobic performance of college students [J]. J Int Soc Sports Nutr. 2019;16(1):18.

28. Borg GA. Psychophysical bases of perceived exertion [J]. Med Sci Sports Exerc. 1982;14(5):377-81. 
29. Camps SG, Wang NX, Tan WS, et al. Estimation of basal metabolic rate in Chinese: are the current prediction equations applicable? [J]. Nutr J. 2016;15(1):79.

30. da Silva RP, de Oliveira LF, Saunders B, et al. Effects of $\beta$-alanine and sodium bicarbonate supplementation on the estimated energy system contribution during high-intensity intermittent exercise [J]. Amino Acids. 2019;51(1):83-96.

31. Bar-Or O. The Wingate anaerobic test. An update on methodology, reliability and validity [J]. Sports Med. 1987;4(6):381-94.

32. Kurtz I, Kraut J, Ornekian V, et al. Acid-base analysis: a critique of the Stewart and bicarbonatecentered approaches [J]. Am J Physiol Renal Physiol. 2008;294(5):F1009-31.

33. di Prampero PE, Ferretti G. The energetics of anaerobic muscle metabolism: a reappraisal of older and recent concepts [J]. Respir Physiol, 1999, 118(2-3): 103 - 15.

34. Ozyener F, Rossiter HB, Ward SA, et al. Influence of exercise intensity on the on- and off-transient kinetics of pulmonary oxygen uptake in humans [J]. J Physiol. 2001;533(Pt 3):891-902.

35. Aoi W, Marunaka Y. Importance of pH homeostasis in metabolic health and diseases: crucial role of membrane proton transport [J]. Biomed Res Int, 2014, 2014: 598986.

36. Ortiz-Prado E, Dunn JF, Vasconez J, et al. Partial pressure of oxygen in the human body: a general review [J]. Am J Blood Res. 2019;9(1):1-14.

37. Rojas Vega S, Strüder HK, Wahrmann BV, et al. Bicarbonate reduces serum prolactin increase induced by exercise to exhaustion [J]. Med Sci Sports Exerc. 2006;38(4):675-80.

38. Calbet JAL, Martín-Rodríguez S, Martin-Rincon M, et al. An integrative approach to the regulation of mitochondrial respiration during exercise: Focus on high-intensity exercise [J]. Redox Biol. 2020;35:101478.

39. Xia S, Chen G, Wang B, et al. Addition of Sodium Pyruvate to Stored Red Blood Cells Attenuates Liver Injury in a Murine Transfusion Model [J]. Mediators Inflamm, 2016, 2016: 3549207.

40. Gou $\mathrm{D}$, Tan $\mathrm{H}$, Cai $\mathrm{H}$, et al. Pyruvate effects on red blood cells during in vitro cardiopulmonary bypass with dogs' blood [J]. Artif Organs. 2012;36(11):988-91.

41. Zhang JJ, Shen HQ, Deng JT, et al. Effect of peritoneal dialysis solution with different pyruvate concentrations on intestinal injury [J]. Exp Biol Med (Maywood). 2020;245(7):644-53.

42. Olivencia-Yurvati AH, Blair JL, Baig $\mathrm{M}$, et al. Pyruvate-enhanced cardioprotection during surgery with cardiopulmonary bypass [J]. J Cardiothorac Vasc Anesth. 2003;17(6):715-20.

43. Yu W, Hu S, Xie ZY, et al. Pyruvate oral rehydration solution improved visceral function and survival in shock rats [J]. J Surg Res. 2015;193(1):344-54.

44. Kane DA. Lactate oxidation at the mitochondria: a lactate-malate-aspartate shuttle at work [J]. Front Neurosci. 2014;8:366.

45. Jones RM, Cook CC, Kilduff LP, et al. Relationship between repeated sprint ability and aerobic capacity in professional soccer players [J]. ScientificWorldJournal, 2013, 2013: 952350. 
46. Robergs R, Kennedy D. Metabolic acidosis and fatigue: Where to from here? [J]. Regulation of Fatigue in Exercise, 2011: 41-61.

47. Crisafulli DL, Buddhadev HH, Brilla LR, et al. Creatine-electrolyte supplementation improves repeated sprint cycling performance: A double blind randomized control study [J]. J Int Soc Sports Nutr. 2018;15:21.

48. Zhou FQ. Pyruvate-enriched fluids as a novel medical solution and beverage [J]. Food Sci \& Nutri Tech, 2021, 6(1): 000258.

\section{Tables}

Table 1 Participant characteristics

\begin{tabular}{|llllllll|}
\hline $\begin{array}{l}\text { Age } \\
\text { (year) }\end{array}$ & $\begin{array}{l}\text { Height } \\
(\mathrm{cm})\end{array}$ & $\begin{array}{l}\text { Weight } \\
(\mathrm{kg})\end{array}$ & $\begin{array}{l}\text { Body fat } \\
(\%)\end{array}$ & $\begin{array}{l}\mathrm{BMl} \\
\left(\mathrm{kg} / \mathrm{m}^{2}\right)\end{array}$ & $\mathrm{VO}_{2 \max }$ & $\mathrm{W}_{\max }$ & $\begin{array}{l}\text { Training year } \\
(\text { year })\end{array}$ \\
\hline $20 \pm 2$ & $\begin{array}{l}178.31 \pm \\
6.36\end{array}$ & $\begin{array}{l}69.58 \pm \\
7.22\end{array}$ & $\begin{array}{l}13.11 \pm \\
3.50\end{array}$ & $\begin{array}{l}21.84 \pm \\
1.33\end{array}$ & $\begin{array}{l}55.93 \pm \\
5.36\end{array}$ & $\begin{array}{l}298.54 \pm \\
41.7\end{array}$ & $9 \pm 3$ \\
\hline
\end{tabular}

Note. Values are mean $\pm \mathrm{SD}$. $\mathrm{BMI}$, body mass index; $\mathrm{VO}_{2 \text { max }}$, maximal oxygen uptake; $\mathrm{W}_{\text {max }}$, maximal power output.

Table 2 Venous blood $\mathrm{pH}, \mathrm{HCO}_{3}{ }^{-}$, $\mathrm{BE}$ and lactate for each group 


\begin{tabular}{|c|c|c|c|c|c|c|}
\hline & Group & Baseline & Pre-HIIE & Post-HIIE & Pre-RSE & Post-RSE \\
\hline \multirow[t]{2}{*}{ Blood pH } & PYR & $\begin{array}{l}7.38 \pm \\
0.04\end{array}$ & $\begin{array}{l}7.40 \pm \\
0.03^{\star \star}\end{array}$ & $\begin{array}{l}7.20 \pm \\
0.05^{\star} \triangle \triangle\end{array}$ & $\begin{array}{l}7.24 \pm \\
0.06 \star \triangle \triangle\end{array}$ & $\begin{array}{l}7.23 \pm \\
0.07 \triangle \triangle\end{array}$ \\
\hline & PLA & $\begin{array}{l}7.36 \pm \\
0.03\end{array}$ & $7.37 \pm 0.03$ & $\begin{array}{l}7.14 \pm \\
0.08 \triangle \triangle\end{array}$ & $\begin{array}{l}7.17 \pm \\
0.09 \triangle \Delta\end{array}$ & $\begin{array}{l}7.18 \pm \\
0.07 \triangle \triangle\end{array}$ \\
\hline \multirow[t]{2}{*}{$\begin{array}{l}\text { Blood } \mathrm{HCO}_{3}{ }^{-} \\
(\mathrm{mmol} / \mathrm{L})\end{array}$} & PYR & $\begin{array}{l}24.55 \pm \\
1.15\end{array}$ & $\begin{array}{l}26.09 \pm \\
0.82^{\star \star \Delta \triangle}\end{array}$ & $\begin{array}{l}14.56 \pm \\
1.83^{\star} \triangle \triangle\end{array}$ & $\begin{array}{l}15.24 \pm \\
2.10^{\star \Delta \triangle \triangle}\end{array}$ & $\begin{array}{l}14.04 \pm \\
2.32 \triangle \triangle\end{array}$ \\
\hline & PLA & $\begin{array}{l}24.52 \pm \\
0.96\end{array}$ & $24.86 \pm 1.03$ & $\begin{array}{l}12.57 \pm \\
1.86 \triangle \triangle\end{array}$ & $\begin{array}{l}13.02 \pm \\
2.05^{\Delta \triangle}\end{array}$ & $\begin{array}{l}12.52 \pm \\
1.61 \triangle \triangle\end{array}$ \\
\hline \multirow[t]{2}{*}{$\begin{array}{l}\text { Blood BE } \\
\text { (mmol/L) }\end{array}$} & PYR & $\begin{array}{l}1.06 \pm \\
1.15\end{array}$ & $\begin{array}{l}3.39 \pm \\
0.86^{\star \star \Delta \triangle \triangle}\end{array}$ & $\begin{array}{l}-12.58 \pm \\
3.24^{\star} \triangle \triangle\end{array}$ & $\begin{array}{l}-11.68 \pm \\
3.49 * \triangle \triangle\end{array}$ & $\begin{array}{l}-13.64 \pm \\
3.50 * \triangle \triangle\end{array}$ \\
\hline & PLA & $\begin{array}{l}1.16 \pm \\
1.30\end{array}$ & $2.00 \pm 1.11$ & $\begin{array}{l}-15.61 \pm \\
4.39 \triangle \triangle\end{array}$ & $\begin{array}{l}-14.98 \pm \\
4.37 \triangle \triangle\end{array}$ & $\begin{array}{l}-16.56 \pm \\
3.07 \triangle \triangle\end{array}$ \\
\hline \multirow[t]{2}{*}{$\begin{array}{l}\text { Blood lactate } \\
\text { (mmol/L) }\end{array}$} & PYR & $\begin{array}{l}2.23 \pm \\
0.61\end{array}$ & $1.69 \pm 0.44$ & $\begin{array}{l}13.58 \pm \\
3.63 \triangle \triangle\end{array}$ & $\begin{array}{l}12.80 \pm \\
2.76 \triangle \triangle\end{array}$ & $\begin{array}{l}14.24 \pm \\
2.81 \triangle \triangle\end{array}$ \\
\hline & PLA & $\begin{array}{l}2.40 \pm \\
0.68\end{array}$ & $2.09 \pm 0.59$ & $\begin{array}{l}14.23 \pm \\
2.56 \triangle \triangle\end{array}$ & $\begin{array}{l}13.42 \pm \\
2.52^{\triangle \triangle}\end{array}$ & $\begin{array}{l}14.70 \pm \\
2.26 \triangle \triangle\end{array}$ \\
\hline
\end{tabular}

Note. Values are mean \pm SD.

Abbreviations: PYR, Group pyruvate; PLA, Group placebo; Pre-HIIE, pre-high-intensity interval exercise; Post-HIIE, post-high-intensity interval exercise; Pre-RSE, pre-repeated sprint exercise; Post-RSE, postrepeated sprint exercise

$*: p<0.05$, compared to PLA; $* *: p<0.01$, compared to PLA; $\triangle: p<0.05$, compared to baseline; $\triangle \Delta: p<$ 0.01 , compared to baseline

\section{Figures}




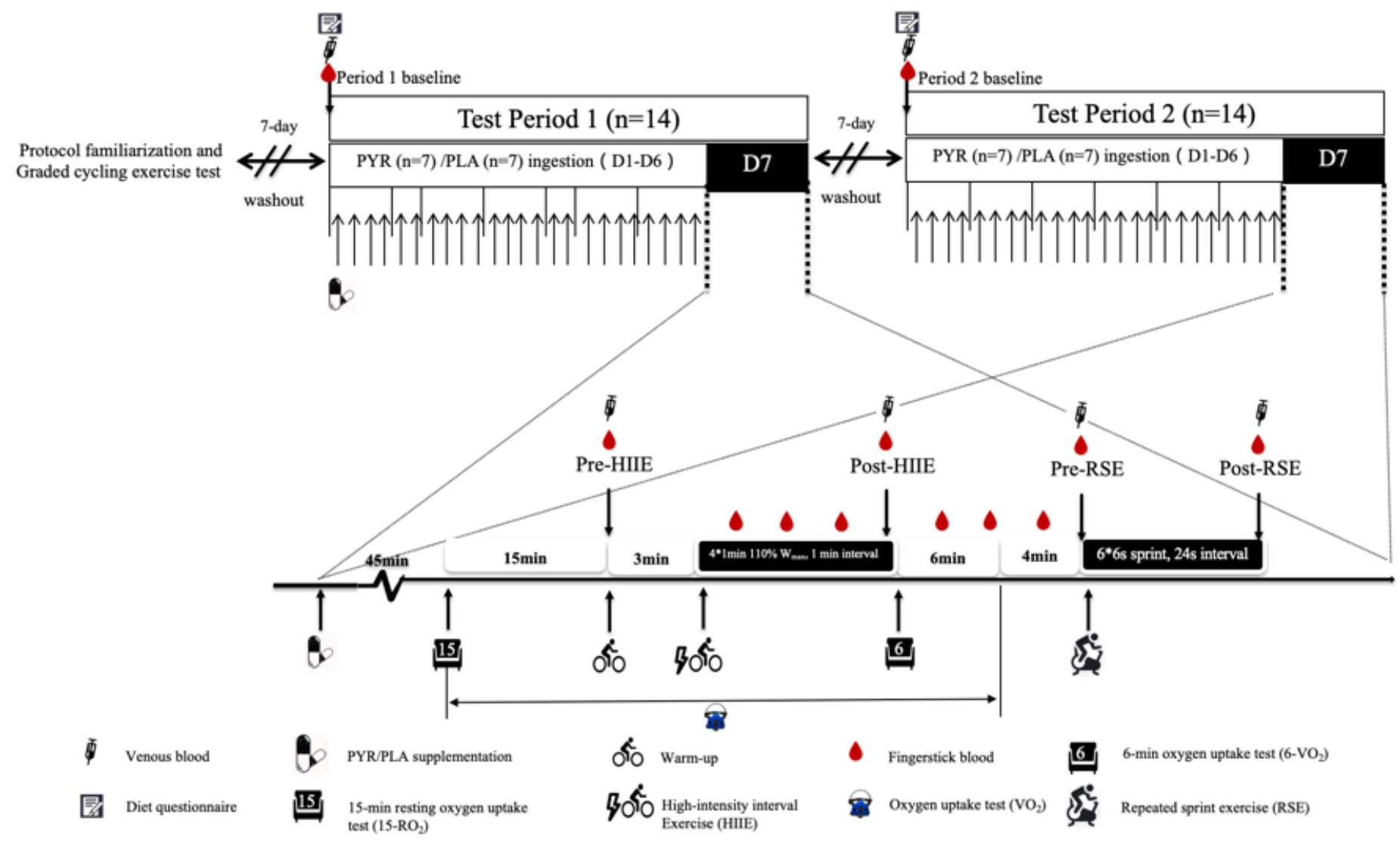

Figure 1

Supplementation and exercise tests timeline 


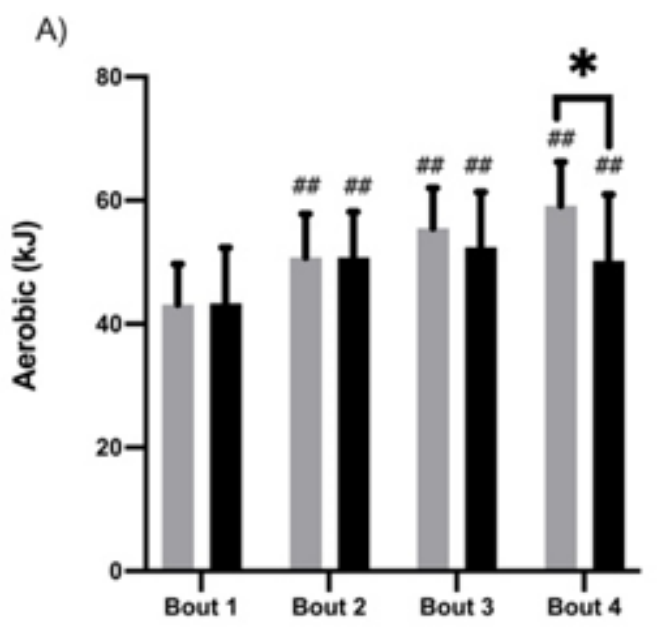

B)
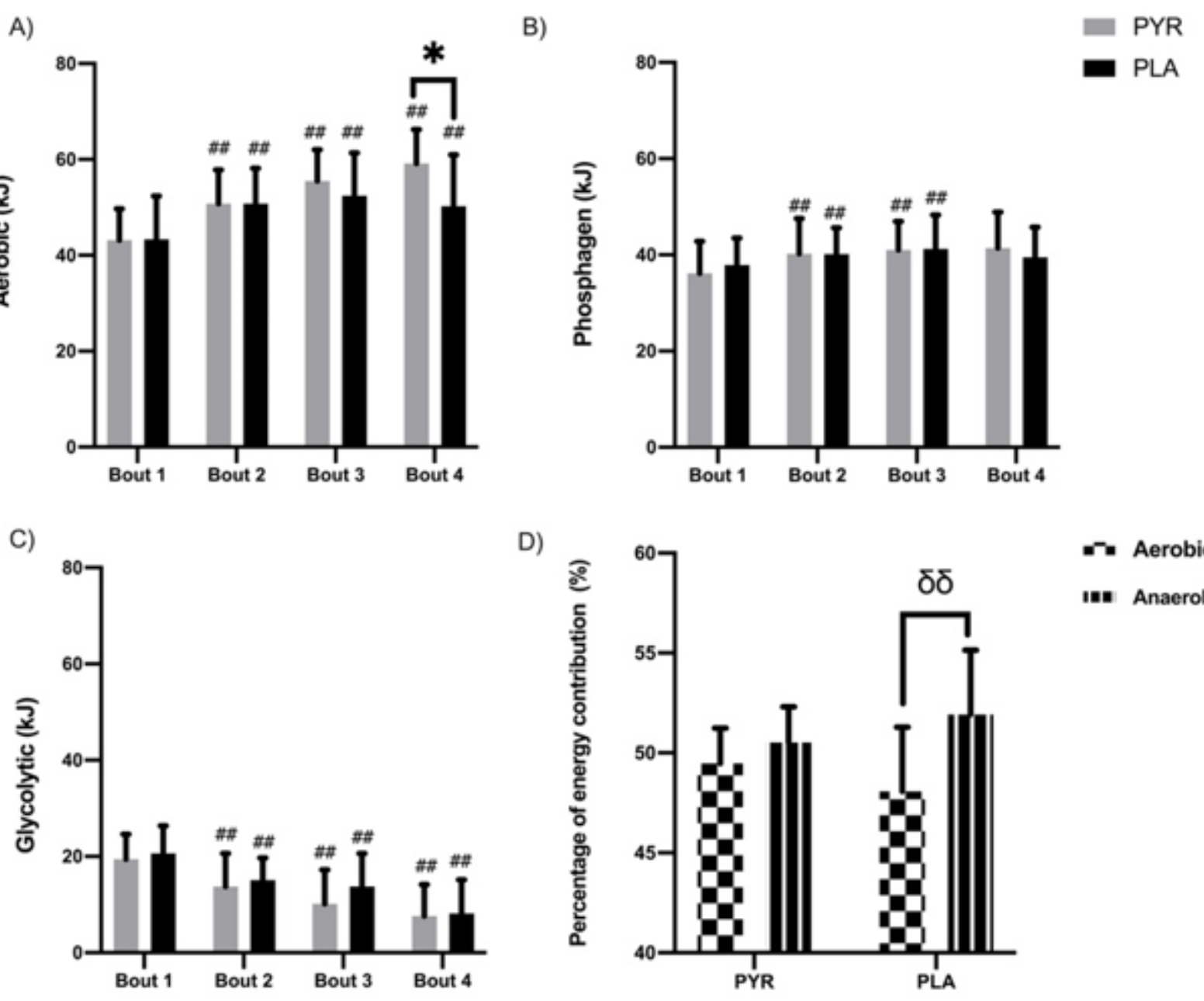

D)

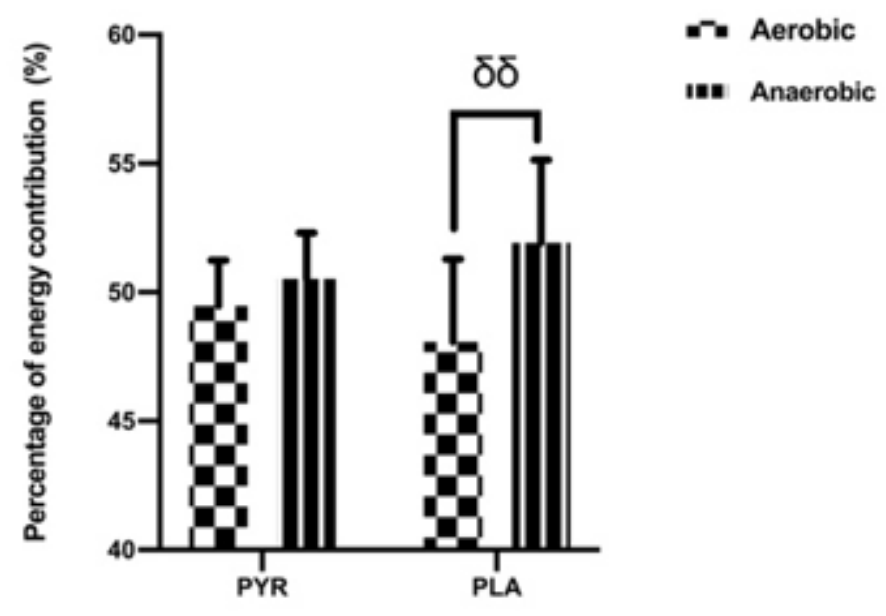

Figure 2

Energy contribution for each group (A-C); the percentage of aerobic and anaerobic energy contributions during HIIE (D). Values are mean \pm SD. PYR, Group pyruvate; PLA, Group placebo; Bout, high-intensity exercise bout; the percentage of aerobic energy contribution = (aerobic energy/total energy) $\mathrm{x} 100$; the percentage of anaerobic energy contribution $=(($ phosphagen energy + glycolytic energy $) /$ total energy $) \mathrm{x}$ $100 ; *$ : $p<0.05$, compared to PLA; \#: $p<0.05$ compared to bout 1 ; \#\#: $p<0.01$, compared to bout $1 ; \delta \delta, p$ $<0.01$, difference between aerobic and anaerobic energy contributions 


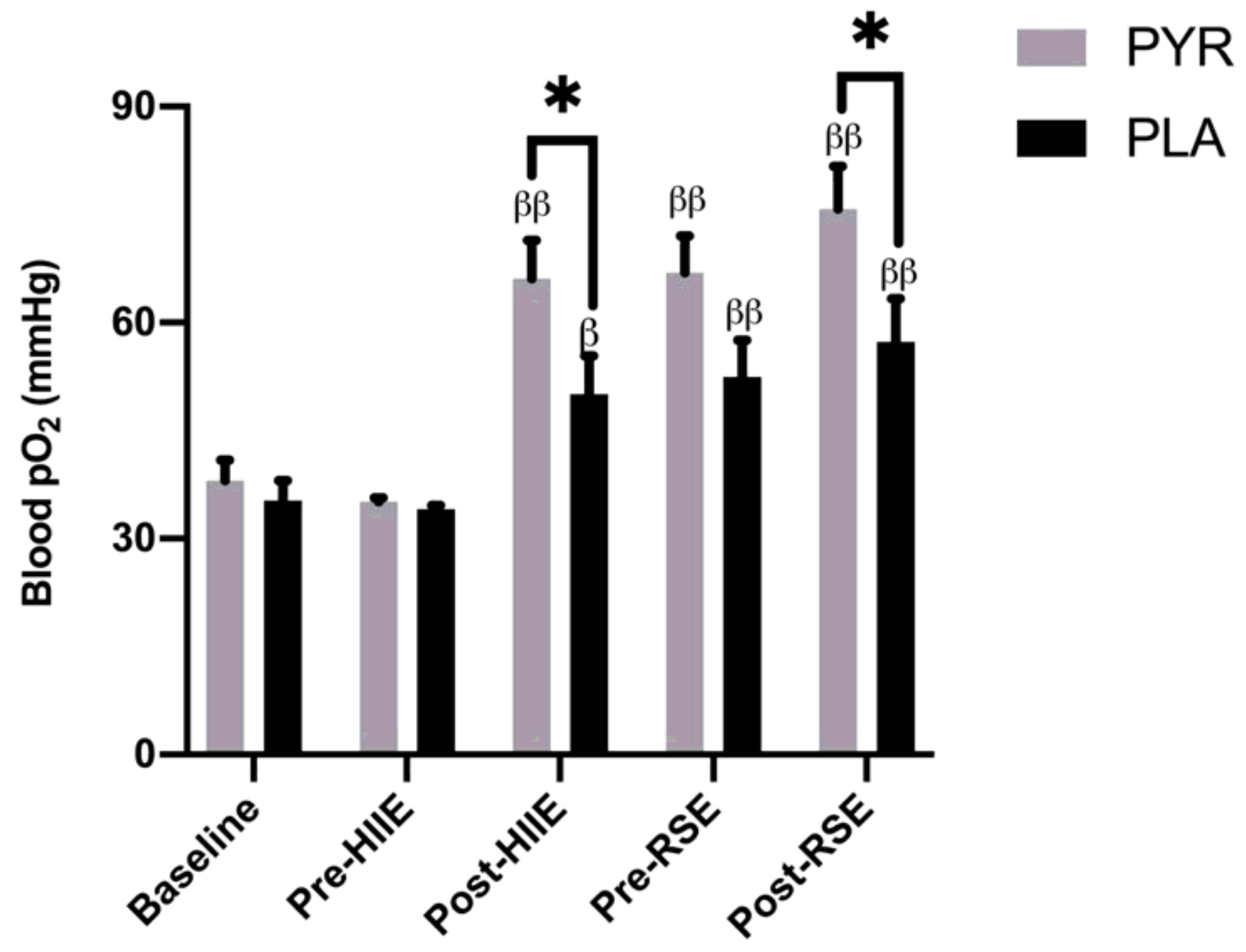

Figure 3

Blood p02 response during tests. Values are mean \pm SD. PYR, Group pyruvate; PLA, Group placebo; PreHIIE, pre-high-intensity interval exercise; Post-HIIE, post-high-intensity interval exercise; Pre-RSE, prerepeated sprint exercise; Post-RSE, post-repeated sprint exercise; *: $p<0.05$, compared to PLA; $\beta$ : $p<0.05$ compared to baseline; $\beta \beta$ : $p<0.01$, compared to baseline 

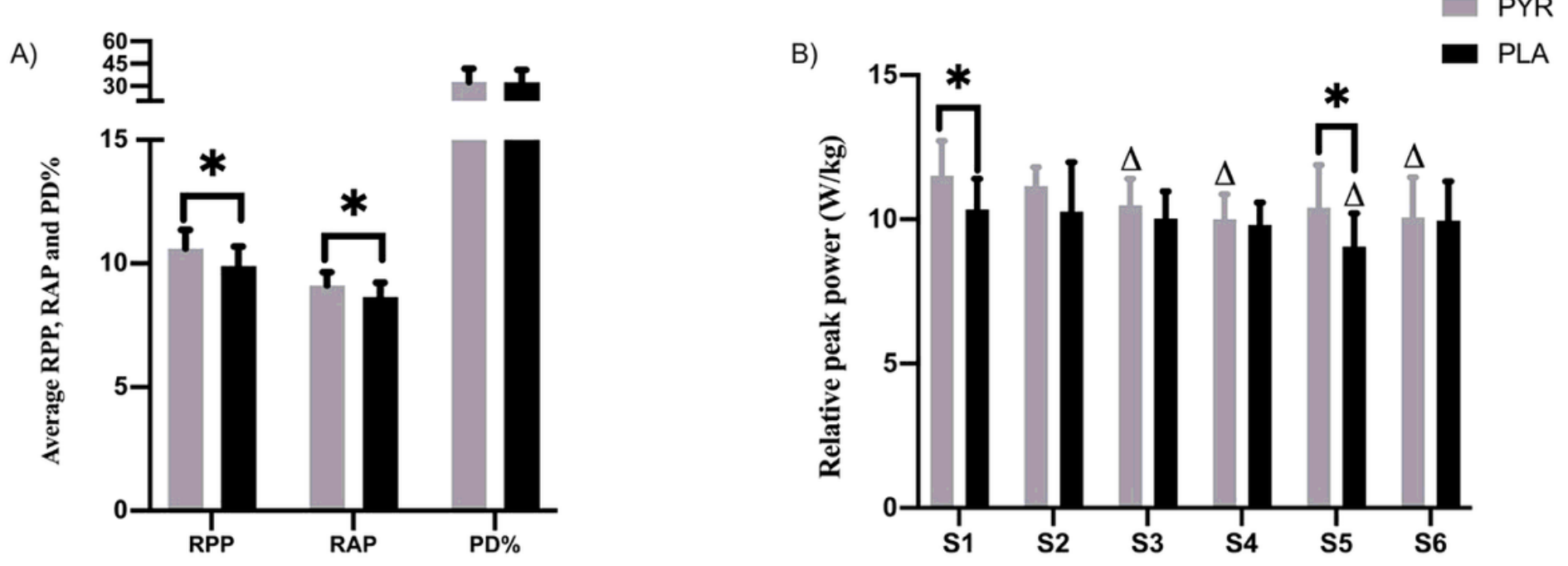

C)
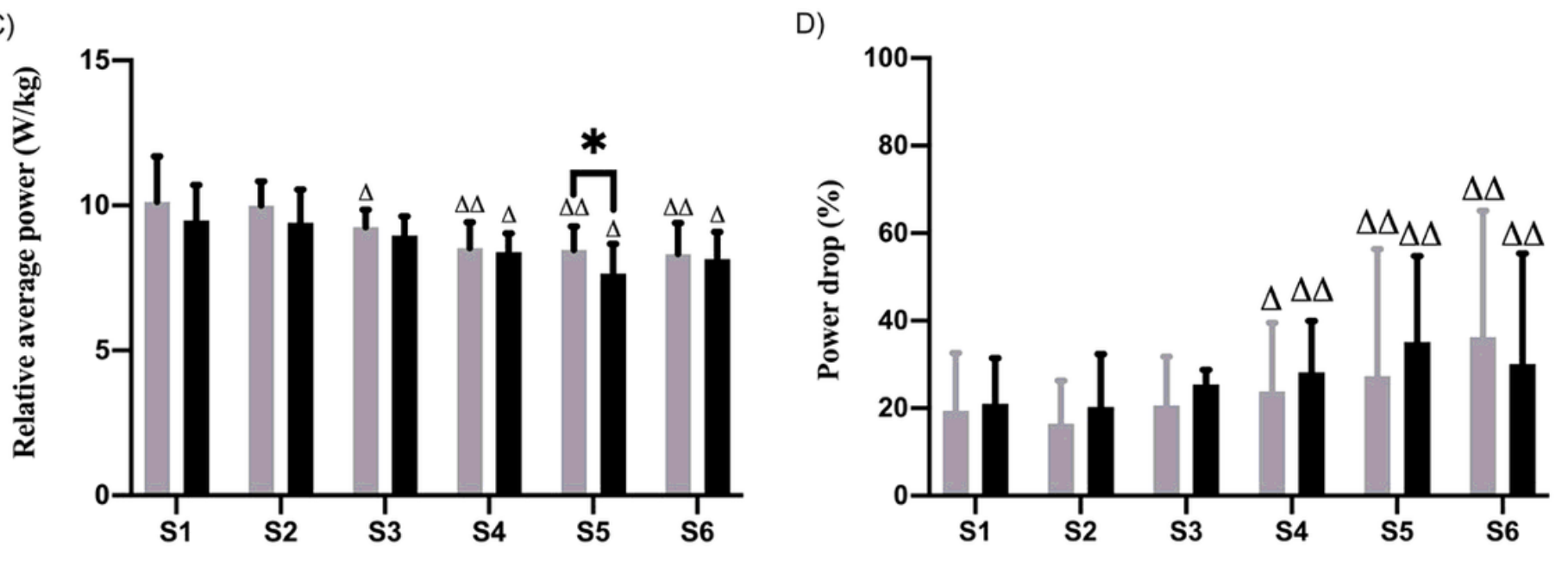

Figure 4

Average RPP, RAP and PD\% during RSE (A). RPP, RAP and PD\% during RSE for each sprint (B-D). Values are mean \pm SD. PYR, Group pyruvate; PLA, Group placebo; S, sprint exercise; RPP, relative peak power; RAP, relative average power; PD\%, power drop; *: $p<0.05$, compared to PLA; $\Delta: p<0.05$, compared to $S 1 ; \Delta \Delta: p$ $<0.01$, compared to S1 